\title{
HEALTH CLOUD IMPLEMENTATION USING SMART ANALYTICS FEATURES
}

\author{
Gaurav Malik ${ }^{1}$, Savita ${ }^{2}$ \\ E-Mail Id: gauravmalik717@gmail.com ${ }^{1}$, savitabamal85@gmail.com² \\ DPGITM Gurugram, Maharshi Dayanand University, Rohtak, Haryana, India
}

\begin{abstract}
Healthcare is undergoing a paradigm shift toward a much greater quality of care delivery. The better care of patients is the driving force behind modernizing many processes; there are plenty of other factors - such as compliance, getting more value out of existing technology investments and workflows implementation, for example, integration of electronic health records (EHR). E-health clouds provide the possibility to easily access the medical data and it provides opportunities to build new business models. It also requires Good coordination and data visualization for the identification of high-risk patients. Many Cloud implementation techniques are made by researchers to collect and utilize patient data effectively. It is important to analyses and identifies the High-risk patient so that prior medications can be done to them. Health Cloud can give healthcare organizations a complete view of patients with rich data from current conditions to medications. It enables a focus on the entire patient history It integrated with EHR and other healthcare systems and get a complete view of the patient's conditions. Einstein Analytics feature on Health Cloud can drive intelligent patient engagement and improve care effectiveness, and manage patient risk. Such type of analysis expert can improve the healthcare industry.
\end{abstract}

Keywords: E-Health Cloud, Healthcare Data Ecosystem, Einstein Analytics.

\section{INTRODUCTION}

The existing healthcare system used in the healthcare sector is facing with several problems like quality, safety, and efficiency of patient care, this problem can be solved by providing a fast way of processing and accessing to the large volume of patient information with integration and effective utilization of data to identify the Risky patient and provide the security of data effectively. With the help of Health Cloud with analytics features, the healthcare organizations can get a complete view of patients in one location with rich data for everything from current conditions to what all the medications needed to patient. When Health Cloud is integrated with EHR and other healthcare systems, it gives a holistic view of the patient and can put the right data in front of the right people. The approach in all these types of systems is to store medical data in some central data. On an international basis, the ISO [9] and the Health Level 7 consortium (HL7) [7] define the standards for e-health infrastructures. Through Einstein Analytics in Healthcare, the care coordinators can utilize critical details and visualize key metrics about their patients. Care coordinators can identify patients who need care plans and take steps to prevent avoidable Risks. Coordinators can segment patients based on different parameters like age, risk barriers to identifying high-risk individuals to help improve their health outcomes. Dashboards also provide insight into the authorization process to help payers.

\section{RESEARCH QUESTIONS}

Considering the existing health cloud limitations and risking demand of patient care, the demand to enhance the health cloud features is rising so that effective patient care is provided, this research demarcates the following research questions (RQ):

RQ1: How to perform Health cloud integration such that Data security can be provided for patient data.

RQ2: How to implement analytics features in Health Cloud to improves care effectiveness to identify high-risk.

\section{LITERATURE REVIEW}

Yan Hu, Fangjie Lu, Israr Khan, Guohua Bai et al. [11] concluded that the superior solution to accomplish the need for healthcare is information sharing in e-health and it can be achieved through the cloud computing. Cloud computing has good performance in storing and accessing information. Their research was focused mainly on the implementation of Software as a Service (SaaS) cloud computing technique such that healthcare information can be shared. They highlighted that the current interoperability gap in e-health could be covered with the designed prototype. The experiment results indicated the use of SaaS service to solve the problems with the help of features the quick development, large data table storage, high availability, application as web service and authorized information.

G.Nikhita Reddy, G.J.Ugander Reddy et al. [12] focused on the advantage of using mobile technologies with cloud technologies as it makes mobility and sharing of the information easier. Cloud Computing based solutions in healthcare can help the doctors to be in touch with their patients and such that their health condition can be effectively monitored at a reasonable cost. The concern regarding the security and other privacy issues of data was highlighted as an open problem 
ICACCG2020 30-31 July, 2020, Ansal University, Gurgaon, India

International Journal of Technical Research \& Science (Special Issue) ISSN No.:2454-2024 (online)

Yan Hu and Guohua Bai et al. [13] presented that research on applying cloud computing technology to eHealth is in its early stages. It was highlighted that most researchers have presented ideas without real-world case validation. The main purpose of their review was to identify the challenges and feasibility of cloud-based solutions such that it can be applied in eHealth. The research review highlighted the superiority of the cloud in its fast processing ability and storage capability using big data technology. The hybrid cloud platform with mixed access control and security protection mechanisms will be the main research area for the healthcare system

Sanjay P Ahuja, Sindhu Mani and Jesus Zambrano et al. [14] focused and decided that the current trend of adopting cloud in the medical field can improve and solve several information issues in healthcare organizations as well as cost optimizations. It was decided that Standardized cloud-based applications can bring more advantages to patients, doctors, pharmacies, insurance companies, etc. When sharing information across medical organizations yielding better results. It was highlighted that the cloud-computing model will have Challenges such as security concerns and interoperability.

Atiya Parveen, Sobia Habib, Waseem Ahmad et al. [15] discussed that the E-health cloud is the next big buzz in the healthcare sector. With the use of cloud computing in the healthcare sector, data can be centralized, and it could best be shared between all the healthcare providers on the cloud, which would allow Good collaboration as well as virtualization. Rural healthcare centers would efficiently use their Cloud infrastructure to increase their profits. The research highlighted that there can be multiple not the one deployment model or service model that can cater to the Healthcare world.

In this paper, it is discussed how to implement a robust and secure health cloud system on the Salesforce platform. Also, it highlighted the Health cloud analytics features and patient data can be integrated in a more intelligent way such that It can provide the metrics about their patients and gives better insights to drive intelligent patient engagement.

\section{RESEARCH METHODOLOGY}

\subsection{Problem Definition}

In the current existing healthcare system, the limited access to the patient data leads to ineffective decision making for patient and decrease the healthcare quality services. The complexity and inflexibility in the existing healthcare system have raised the requirement of the effective Heath cloud with analytics and continuous integration features

\subsection{Objectives}

The goal of this research paper can be split up as follows.

\subsubsection{Concept}

Understand the concept of Health cloud on the Salesforce platform.

\subsubsection{Method}

After the initial research, select methods that provide continuous integration Health cloud with an external system using Einstein analytics features.

\subsubsection{Evaluate}

Patient data on the cloud is analyzed so that it lets to identify high-risk patients. This information is used to manage those high-risk patients such that preventive care can be done. Multiple embedded dashboards that are connected with the Heath Cloud risk scoring tables that are the results from the predictive analysis of health cloud data are useful in making intelligent decisions.

\subsection{Research Design}

Designing a Health cloud includes below integrated components.

\subsubsection{Objects}

Health Cloud information is stored in the form of standard and custom objects. API calls are used verification of custom objects and fields Objects Represent database tables, for example, a central object represents the organization

Some objects are associated with Health cloud associated object i.e. Feed objects. It is with associated with standard objects through Standard Object Name Feed model, Belo are different functions to manage the supported calls describes Objects(), get Deleted(), delete(), get Updated(), query().

\subsubsection{Patient Activation on Cloud}

Once patients imported into Health Cloud, the information required to identify and represent those patients is created. Custom integration is used to import medical records including historical data from the EHR system and it can be automated with the help of default patient creation job flow. The patient is represented in Health Cloud as a candidate for services so patient object starts with a Candidate Patient record. Based on the mapping of fields 
ICACCG2020 30-31 July, 2020, Ansal University, Gurgaon, India

International Journal of Technical Research \& Science (Special Issue) ISSN No.:2454-2024 (online)

between Candidate patient and the Salesforce object the data is copied to the Salesforce objects, Once the candidate patient is converted, the patient creation process creates corresponding objects in Salesforce:

\subsubsection{Electronic Health Record}

EHRs are the patient real records that make instant information available to authorized users. EHR contains the medical and treatment histories of the patient. EHR systems collect the standard clinical data in a provider's office and that data provides a broader view of a patient's care HL7 for exchanging electronic health records (EHR). The EHR data transmitted via HL7, the (ADT) message stores patient information in the Salesforce Health Cloud data model. Data elements ADT messages contains Data element and these data elements are stored in the Salesforce Account and Contact objects and in Health Cloud custom objects. Elements are listed here in order of sequence numbers in the HL7 standard.

\subsubsection{Health Cloud APIs}

Health Cloud Business APIs are created for integrations with Health Cloud or for creating custom UI components. With the help of API wrap business, the uses cases for the healthcare industry are implemented as highlighted below

\subsubsection{Patient Record Creation}

Patient record creation is done with the help of API calls, it is done with the help of Apex code that is similar to class and object relationship feature as defined below

public class Healthcloud

\{

public static void Patient create()

Health Cloud GA. Patient patient $=$ new Health cloud Patient create ();

patient. Patient Class = 'Individual';

patient. First Name = 'ABC';

Health Cloud GA. Result res = Health Cloud GA. Patient Service. Create Patient(patient);

System.debug(res);

\}

4.3.4.2 Patient Enrollment to Care Program Using REST API: Health Cloud Business APIs can be accessed using REST API design methodology REST API extents HTTP methodology, it uses GET methods to retrieve the resources and PUT methods to update the resource. RESTful API is helpful in Cloud services as service binding through API can be easily controlled by controlling URL Below is the REST API Example:

\subsubsection{Resources}

Health Cloud Business APIs is integrated with other applications with the help of Rest API framework below is the sample for enrollments endpoint URL used for patient care-programs enrollment endpoint url: /services/data/vXX.X/connect/health/care-programs/enrollments.

4.3.4.4 Request Bodies: This section lists the request bodies for Health Cloud Business APIs.

resource.

Root XML tag

<careProgramEnrollmentData>

JSON body

\{

"programId": "programId1",

"sourceSystem": "source-system-name",

"patientData":

I

\{

"patientId": "patient1",

"careProgramProductData":

[

\{

"providerId": "provider1",

\}

"productId": "product1"

]

\}

]

\}

4.3.4.5 Response Bodies: The Response in JSON format contains patient information.

DOI Number: https://doi.org/10.30780/specialissue-ICACCG2020/003

Paper Id: IJTRS-ICACCG2020-003

pg. 16

@ 2017, IJTRS All Right Reserved, www.ijtrs.com 
ICACCG2020 30-31 July, 2020, Ansal University, Gurgaon, India

International Journal of Technical Research \& Science (Special Issue) ISSN No.:2454-2024 (online)

\section{ANALYSIS AND FINDINGS}

In this section, the results of the implementation of Patient data on the cloud is analyzed so that it lets hospitals identify high-risk patients. This information is used to manage those high-risk patients such that preventive care can be done. Multiple embedded dashboards that are connected with the Heath Cloud risk scoring tables that are the results from the predictive analysis of health cloud data, that helps in making intelligent decisions. Analysis and findings are highlighted below in the form of a dashboard representation of data.

\subsection{Risk Stratification}

Risk Stratification is used to identify high-risk patients. The information taken as an output from the risk adjustment model is used to identify patients with high Risks. The embedded dashboards are connected with the risk scoring tables of Heath Cloud. Risk scores based on the CMS-Hierarchical Condition Category risk adjustment model as shown in Fig-5.1. is calculated and with the help of the Risk Scoring Algorithm Patient Summary record is generated and analyzed. The algorithm job runs and in case there is a change in underlying records, the system generates a new Patient Summary record, so that help in analyzing risk data that alert the Doctors about those patients who fall into a particular population, such as the risk for admission, diagnostics or probability of a serious disease like Covid-19.

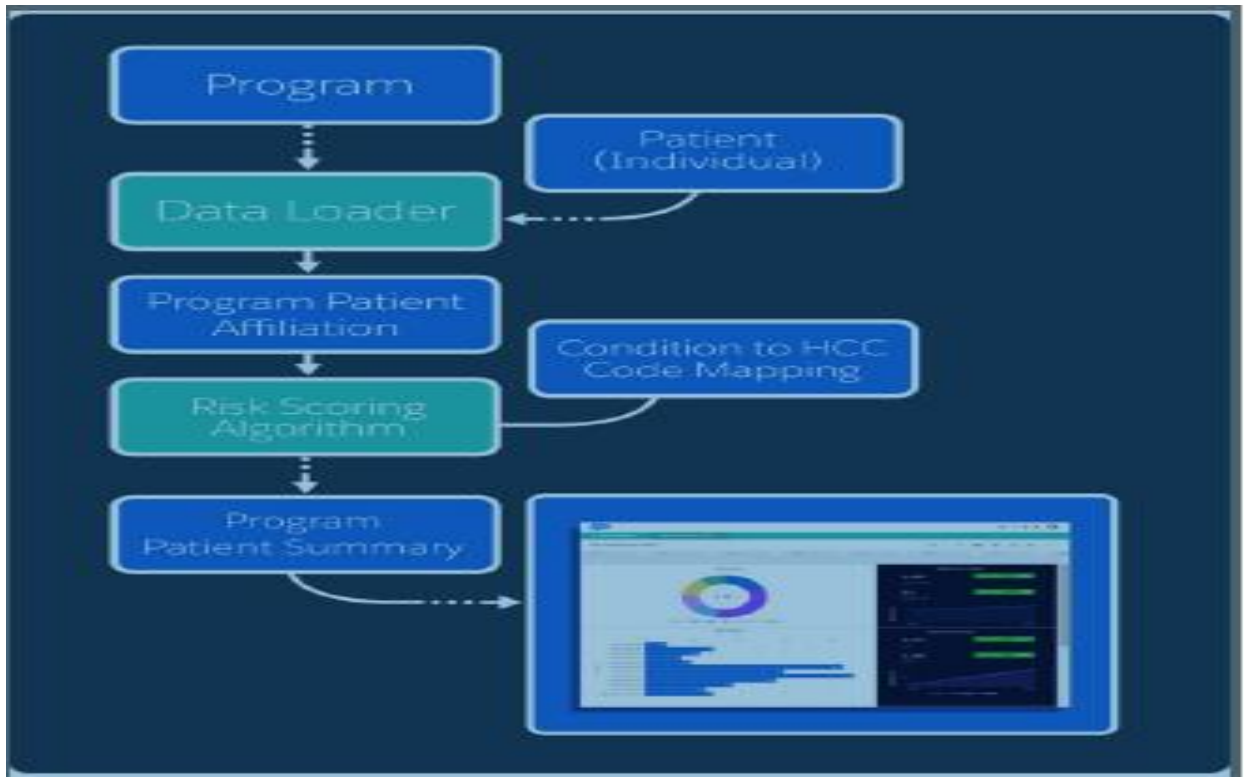

Fig-5.1 CMS-Hierarchical Condition Category (HCC) Risk Adjustment Model

\subsection{Patient Life Event Analysis}

The Person Life Event gives a view of a patient's life events that help them by identifying the Risk and help them by deciding the type of care plan tailored to the patient's condition and life events. As highlighted below Patient Life event can be tracked and the decision can be taken based on visualized Result It can show the Life Events personal or professional that may have impacted the health of the patient as shown in Fig-5.2.

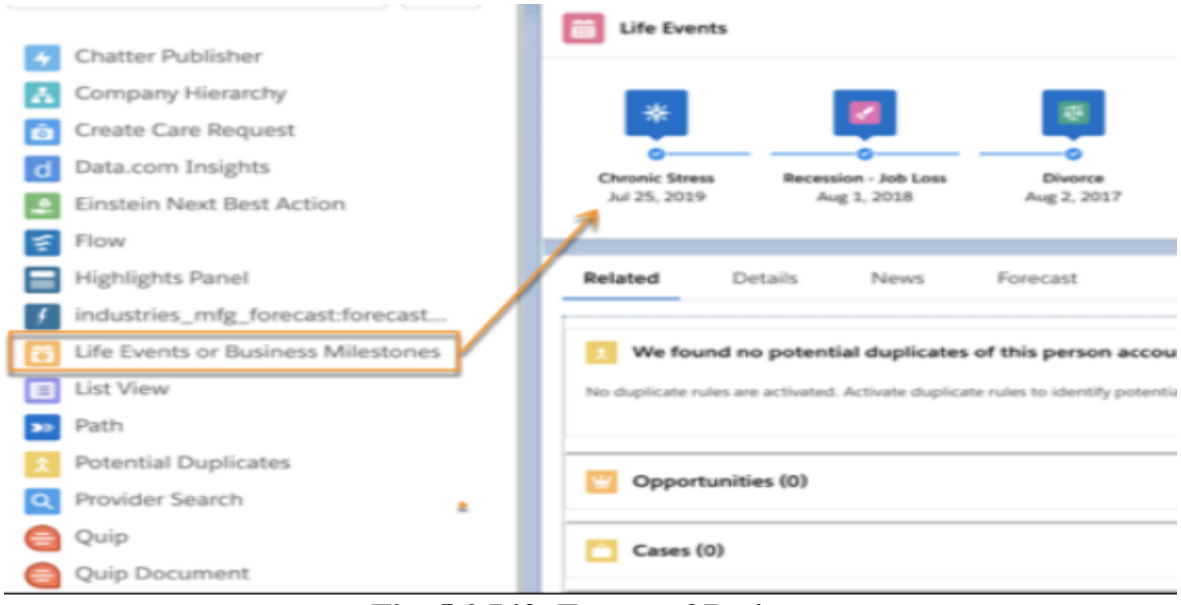

Fig. 5.2 Life Events of Patient

DOI Number: https://doi.org/10.30780/specialissue-ICACCG2020/003

Paper Id: IJTRS-ICACCG2020-003

pg. 17

@ 2017, IJTRS All Right Reserved, www.ijtrs.com 
ICACCG2020 30-31 July, 2020, Ansal University, Gurgaon, India

International Journal of Technical Research \& Science (Special Issue) ISSN No.:2454-2024 (online)

\subsection{Care Co-Ordination}

The dashboard gives the summarized view of data that can help care coordinators to optimize their workload with dashboards. The data from source reports are represented in the form of different dashboards which can be charts, gauges, tables, metrics. Different types of data can be visualized through the dashboard as highlighted below:

\subsubsection{Care Plan Status}

It displays the total no of the current care plan currently opted by the patient or future care plan that going to come for Patients as shown in Fig. 5.3 Care plan dashboard.
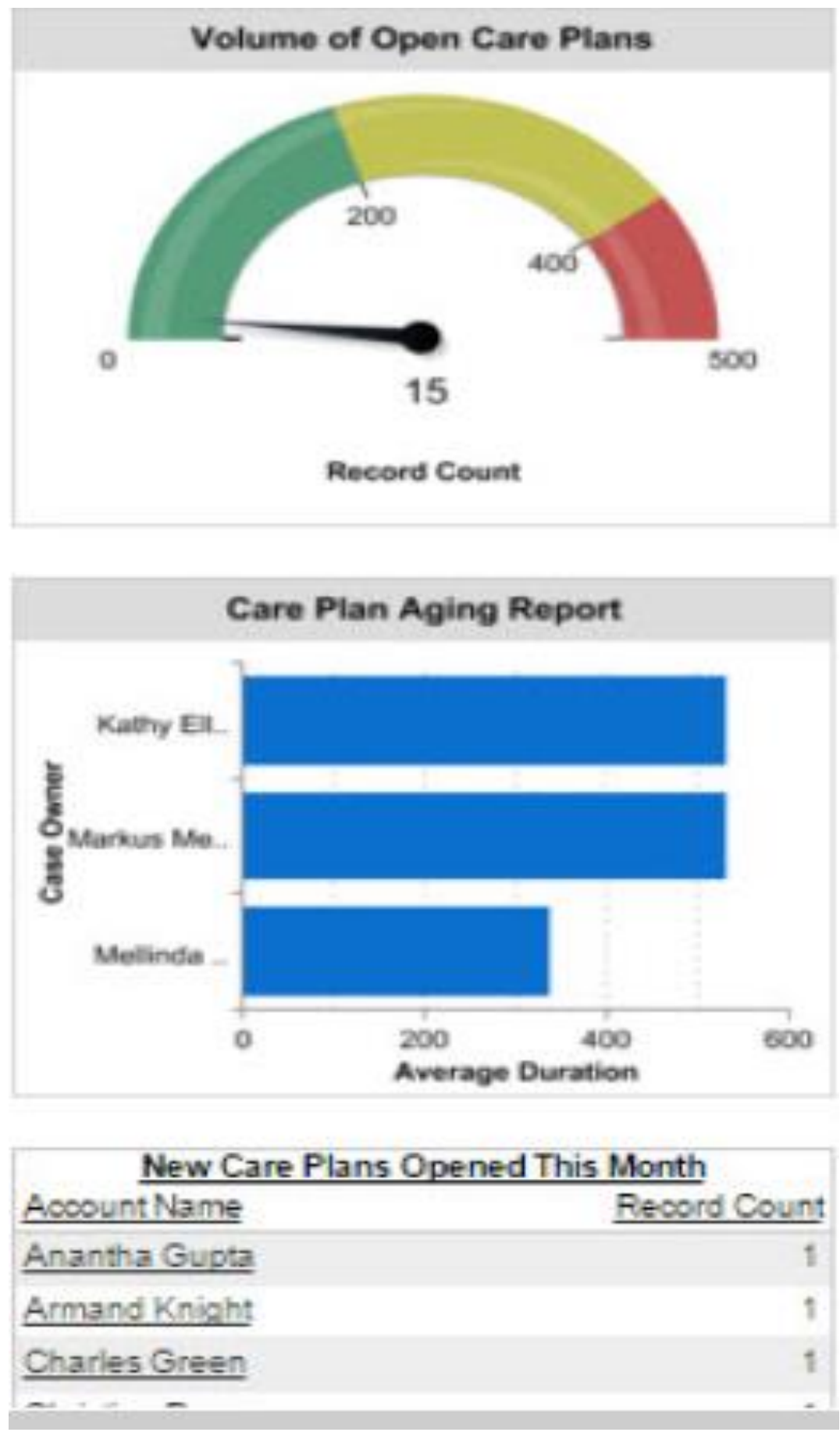

Fig. 5.3 Care Plan Dashboard

\subsubsection{Disease Statistics and Medication Needed}

This Dashboard will show the details of medications prescribed in future again disease, it also displays other parameters like duration of consultation needed as per disease as shown in Fig. 5.4.

DOI Number: https://doi.org/10.30780/specialissue-ICACCG2020/003

pg. 18 
ICACCG2020 30-31 July, 2020, Ansal University, Gurgaon, India

International Journal of Technical Research \& Science (Special Issue) ISSN No.:2454-2024 (online)
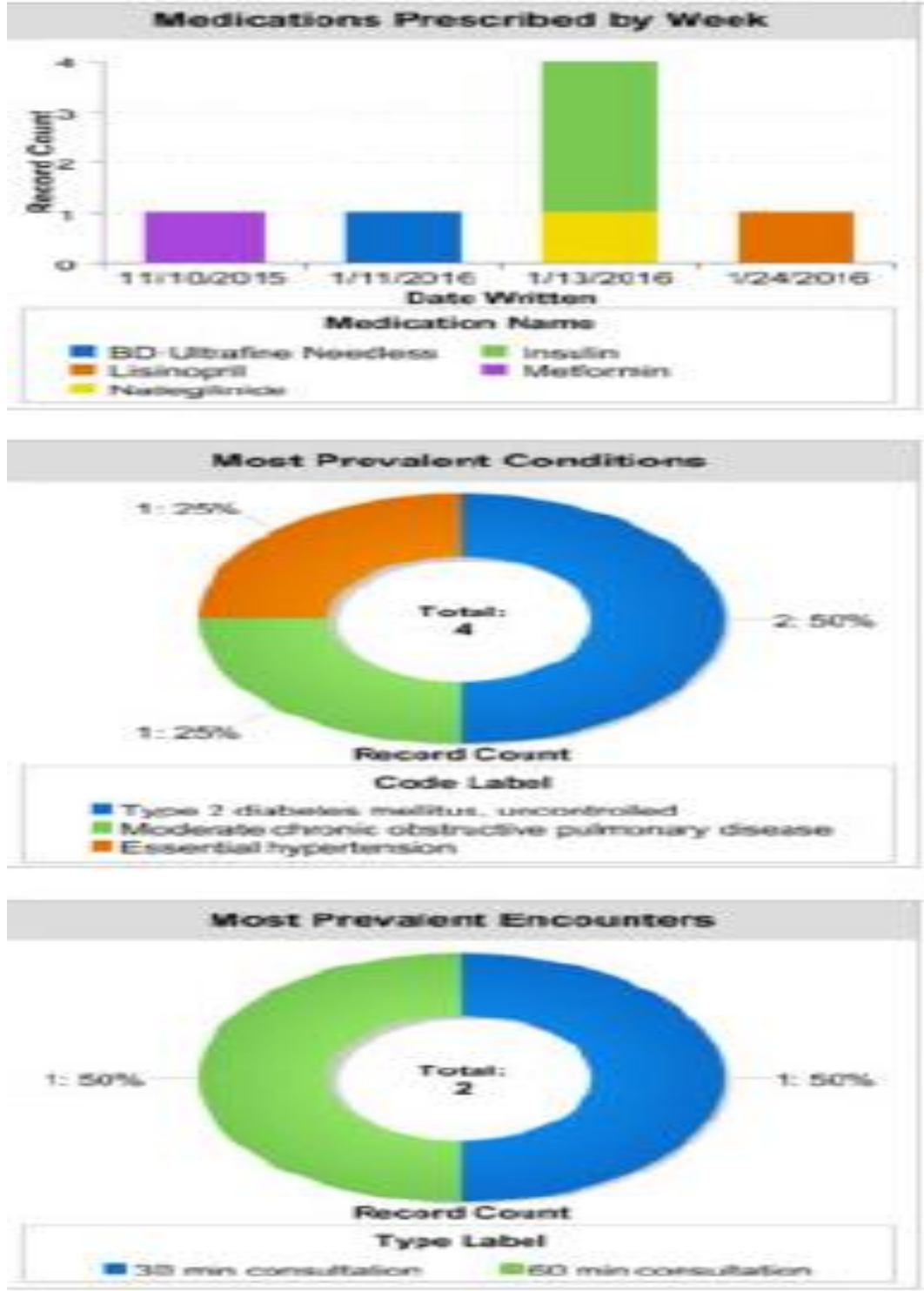

Fig. 5.4 Medication and Disease Dashboard

\section{DISCUSSION AND IMPLICATIONS}

In the past, health care providers used to store medical records of their patients on paper locally. Due to which there was a chance of data privacy and security: But nowadays due to cloud computing services all data of the patient can be stored in the cloud and managed for analysis, in this paper, we discuss the implementation of Health Cloud with analytics features such that it connects to patients in the personal, 1-to-1 way they expect, integrating these independent modules. In this section, different level of Integrations in Health Cloud is discussed as highlighted below.

\subsection{Security Integration}

Security Integration is the mechanism to authenticate the users and prove the security to patient data. Security Integration keeps checks on client applications such that it log in using valid credentials and are it is subjected to the same security protections that are used in the Salesforce user interface. Security in the Health cloud is constantly developing below are the key features.

\subsubsection{Protect Your Health Data with Salesforce Shield}

Salesforce Shield can use regulations on storing sensitive health information. With the help of this feature the malicious activity and data protection can be done.

\subsubsection{Platform Encryption}

Health cloud is encrypting most sensitive data using Platform Encryption, it addresses the HIPAA requirements for storing sensitive protected health information. Encryption helps you protect PHI, sensitive and proprietary data. It 
ICACCG2020 30-31 July, 2020, Ansal University, Gurgaon, India

International Journal of Technical Research \& Science (Special Issue) ISSN No.:2454-2024 (online)

enables internal and external and data compliance also controls the encryption keys data permissions to protect from unauthorized users.

\subsubsection{Event Monitoring}

Event Monitoring provides access to security and usage data on Salesforce apps such that each interaction can be tracked and accessed via API also, data can be easily imported into any data visualization or monitoring tool.

\subsection{USER INTERFACE INTEGRATION}

User interfaces (UI) of two or more apps can help to create composite apps. This approach provides a single point of entry into multiple applications Health Cloud supports the standard data model as shown in Fig. 6.1. that helps the patients or members information can be represented using Person accounts.

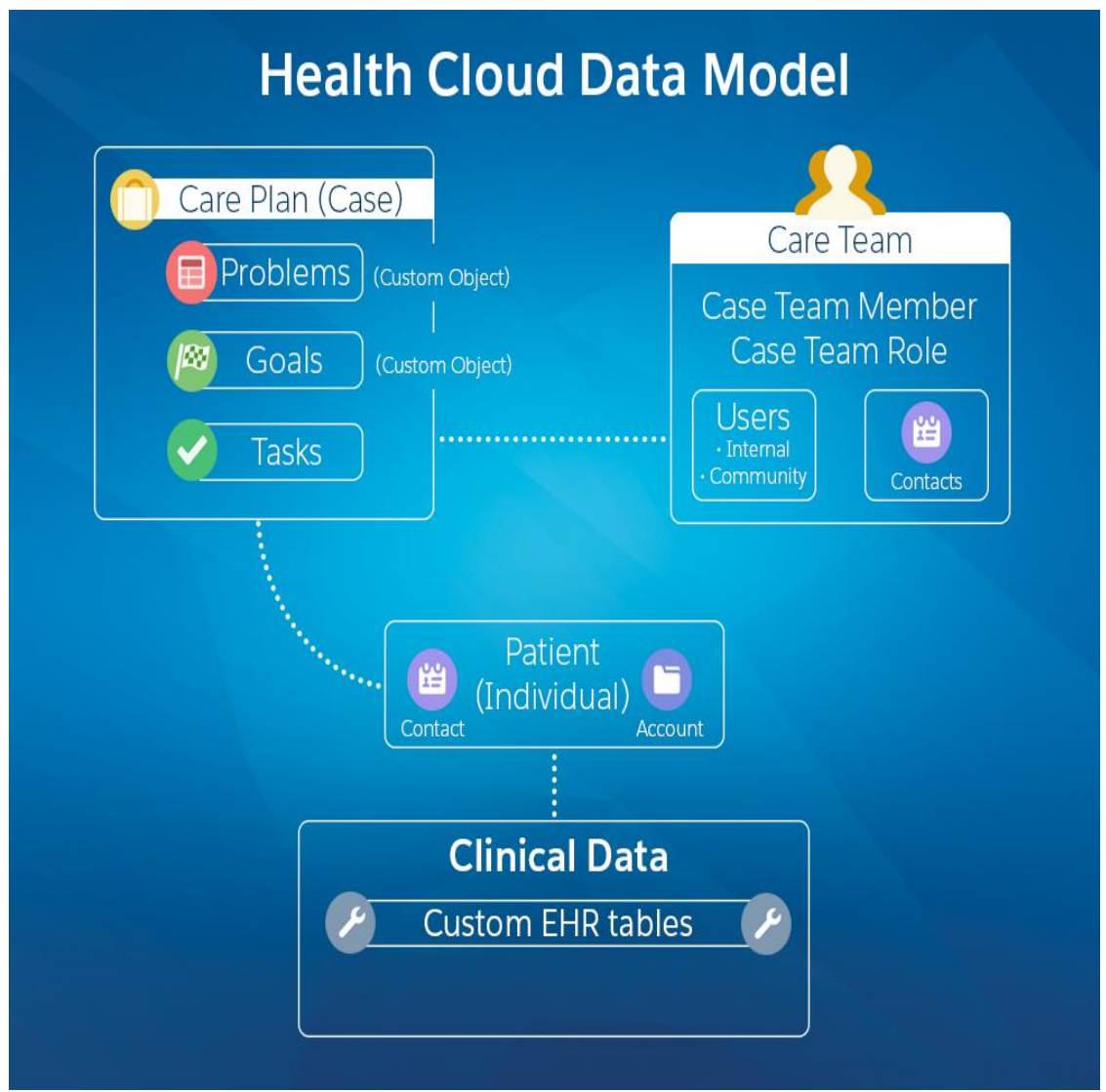

Fig. 6.1 Health Cloud Data Model

\subsection{Business Logic Integration}

With the help of Business logic, one app to another app can be integrated to implement end-to-end business processes. Business logic integration can be easily done through the Salesforce Health Cloud. An important first step is to clearly define your own organization's objectives and expected outcomes. Below are the features that customers provided by Health Cloud.

$>$ Aggregating data.

$>$ Provide a single view.

$>$ Integrate data with analytics software to deliver key insights to the right people.

$>$ Make data accessible anywhere, anytime.

One app to another complete end-to-end business process is done with the help of Apex Web Services calls i.e. Callouts, Email Services, and Outbound Messaging. Apex code is integrated with external service with the help of apex callout.

\subsubsection{Inbound Logic Integration}

Apex Web Services: You can expose Apex class methods as Web services, a feature that provides a way for external applications to execute Apex supports both RESTful and SOAP Web services. Though email services the Apex classes can process the contents and of inbound email.

\subsubsection{Outbound Logic Integration}

Apex Callouts is the feature that integrates Apex code with an external service by a call to an external Web service Apex provides integration with Web services through SOAP and WSDL. Any kind of change in the data is triggered 
ICACCG2020 30-31 July, 2020, Ansal University, Gurgaon, India

International Journal of Technical Research \& Science (Special Issue) ISSN No.:2454-2024 (online)

automatically through Workflow rules once field changes in data are done and trigger automatically the Outbound messaging

\subsection{DATA INTEGRATION}

Through data integration, the view of patients can be seen from current health conditions to medications needed in one location It enables a focus on the entire patient history, not just a visit-by-visit experience. Health Cloud and EHR are integrated with healthcare systems such that it gives a view of the patient and the right data can be presented in front of the right people as shown in Fig. 6.2. The platforms of the health care provider interconnect the smartcard reader and the telematics infrastructure through connectors. The connector is connected to other networks that which are not part of the telematics system [8].

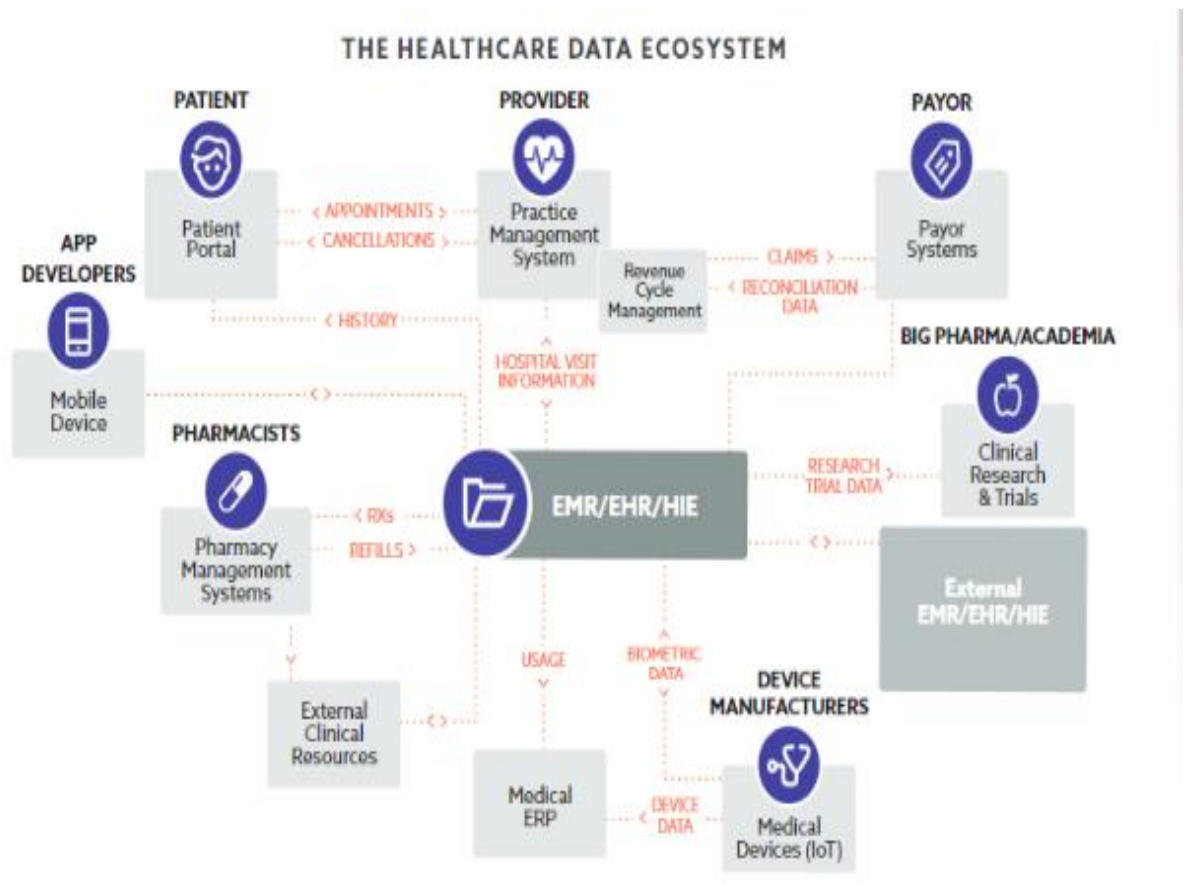

Fig. 6.2 Healthcare Data Ecosystem

The data model allows clinical data integration from other systems in a straightforward and efficient way. The message from EHR system is integrated into the correct Health Cloud object. Patient data is automatically replicated into the data model.

Below are a few Health Cloud objects to manage patient data.

\subsubsection{Account}

In Health Cloud, the account record represents people. The Account object maintains the transactions that occur related to the patient.

\subsubsection{Contact}

In the Salesforce data model, contacts are the people associated with the patient, such as family members and specialists who are outside of your organization.

\subsubsection{User}

It includes internal Salesforce users and community users different access on user types.

\subsubsection{Problems}

Each care plan has a list of clinical or non-clinical health issues that must be addressed.

\subsubsection{EHR Clinical Data Objects}

This custom object holds patient data that comes from the EHR system of record. For example,

EHR Condition represents information about conditions, problems also in case of diagnosis is recognized by a clinician.

\subsection{Analytics Integration}

Einstein Analytics for Health Cloud provides Customer Intelligence Solution. It provides patient engagement in a more intelligent way such that it improves care effectiveness and helps in managing the patient risk much 
ICACCG2020 30-31 July, 2020, Ansal University, Gurgaon, India

International Journal of Technical Research \& Science (Special Issue) ISSN No.:2454-2024 (online)

effectively. It provides metrics about their patients and gives payers and providers the insights to drive intelligent patient engagement. The visualizations of Health Cloud metrics can be used to improve the effectiveness and efficiency of patient care. The coordinators can segment patients based on age, risk, and barriers to identify high-risk patients such that helps in improving health outcomes. With the help of Risk Stratification, high-risk patients can be identified. Though this process patient data integrated with Health Cloud tables such that it aggregates and calculates patient risk. It provides that Mappings that used to calculate risk scores for different age groups of patients. For example, patients who are between 40-45 years old and patients who are 50-55 are in two different age bands. Einstein Analytics for Health Cloud use the mappings of ICD condition and HCC codes to calculated patient risk External data can be integrated using API, the upload external data files for Analytics. A data file that contains external details is in CSV format. The data objects are used to load the data in multiple smaller chunks. The dataflow job is created and marked active and when the file upload is complete status can be monitored, so we can trigger the upload process and also request the cancelation and cleanup process when needed. Once data is uploaded, we can perform the analysis, smart notifications, and dashboard analysis on patient data. End to End Automation flow of data from external system is shown in Fig. 6.3.

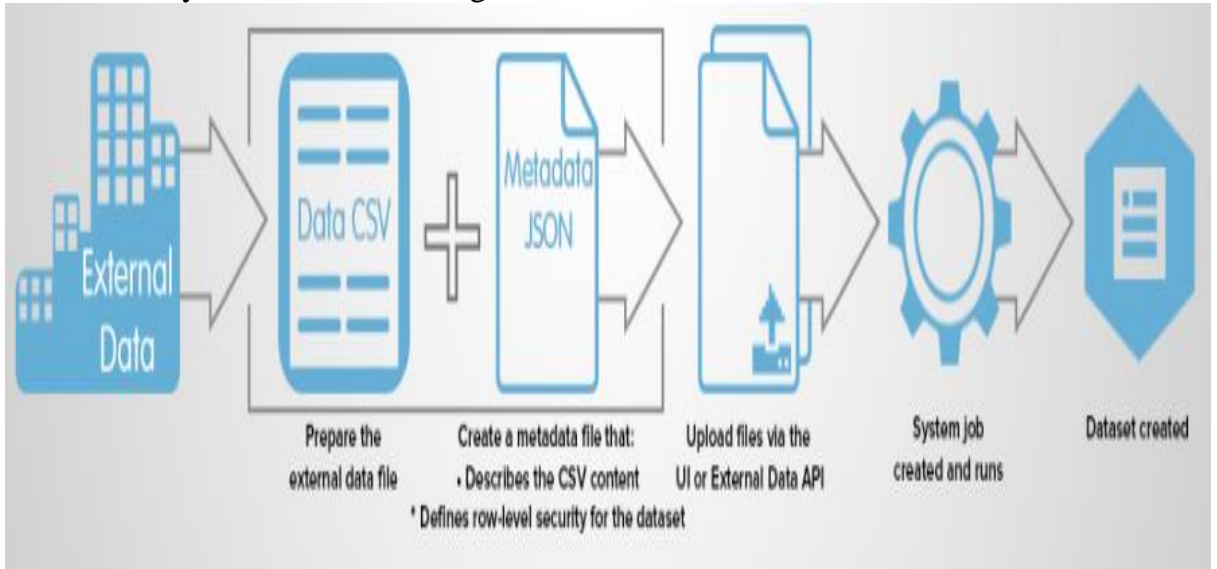

Fig. 6.3 Data Extraction Process

\section{RECOMMENDATIONS AND DIRECTION FOR FURTHER RESEARCH}

In this paper, we discuss security issues in Health cloud and discussed the analytical approach in health cloud for effective Integration of EHR data with Health Cloud such that it can unlock a panoramic view of each patient, Hence, this work can be useful to succeed in an evolving India healthcare industry, providers must build personalized, engaging relationships with each patient. It also enables the doctor or cares coordinator to take proactive steps to reduce the risk of a more serious health incident such that, the risk of a Patient's life, hence that can be managed effectively based on the Health cloud with analytics.

\section{REFERENCES}

[1] P. Barham, B. Dragovic, K. Fraser, S. Hand, T. L.Harris, A. Ho, R. Neugebauer, I. Pratt, and War_eld. Xen and the art of virtualization. In $19^{\text {th }}$ ACM Symposium on Operating Systems Principles (SOSP'03), pages 164\{177. ACM Press, 2003.

[2] S. Berger, R. C_aceres, D. E. Pendarakis, R. Sailer,

[3] E. Valdez, R. Perez, W. Schildhauer, and D. Srinivasan. TVDc: Managing security in the trusted virtual datacenter. Operating Systems Review, 42(1):40-47, 2008.

[4] A. Bussani, J. L. Gri_n, B. Jansen, K. Julisch,G. Karjoth, H. Maruyama, M. Nakamura, R. Perez, M. Schunter, A. Tanner, L. V. Doorn, E. A. V.Herreweghen, M. Waidner, and S. Yoshihama. Trusted Virtual Domains: Secure foundations for business and IT services. Technical Report RC23792, IBM Research, 2005.

[5] S. Cabuk, C. I. Dalton, K. Eriksson, D. Kuhlmann, H. V. Ramasamy, G. Ramunno, A.-R. Sadeghi, M. Schunter, and C. Stuble. Towards automated security policy enforcement in multi-tenant virtual data centers. Journal of Computer Security,18(1):89-121, 2010.

[6] L. Catuogno, A. Dmitrienko, K. Eriksson, D. Kuhlmann, G. Ramunno, A.-R. Sadeghi, S. Schulz,M. Schunter, M. Winandy, and J. Zhan. Trusted Virtual Domains \{ design, implementation and lessons learned. In International Conference on Trusted Systems 2009 (INTRUST'09). Springer Verlag, 2009.

[7] L. Catuogno, H. Lohr, M. Manulis, A.-R. Sadeghi, C. Stuble, and M. Winandy. Trusted Virtual Domains: Color your network. Datenschutz und Datensicherheit (DuD), 5, 2010.

[8] Health Level Seven International (HL7).http://www.hl7.org

[9] Gematik. Einführung der Gesundheitskarte -Gesamtarchitektur, Version 1.7.0. http://www.gematik.de/upload/GA_ZentraleDienste_5171.zip,August 2009. 
ICACCG2020 30-31 July, 2020, Ansal University, Gurgaon, India

International Journal of Technical Research \& Science (Special Issue) ISSN No.:2454-2024 (online)

[10] International Organization for Standardization (ISO).Technical Committee 215, Health Informatics. http://www.iso.org/iso/iso_technical_committee?commid=54960.

[11] Salesforce Health Cloud. https://www.salesforce.com/solutions/industries/healthcare/health-cloud/

[12] Yan Hu, Fangjie Lu, Israr Khan, Guohua Bai, A Cloud Computing Solution for Sharing Healthcare Information, The 7th International Conference for Internet Technology and Secured Transactions (ICITST), IEEE, 2012, London.

[13] G.Nikhita Reddy, G.J.Ugander Reddy, Study of Cloud Computing in HealthCare Industry.

[14] Yan Hu and Guohua Bai, A systematic literature review of cloud computing in e-health, Health Informatics, An InternationalJournal (HIIJ) Vol.3, No.4, pp.11-20, November 2014.

[15] Sanjay P. Ahuja1, Sindhu Mani1 \& Jesus Zambrano1, A Survey of the State of Cloud Computing in Healthcare, Network and Communication Technologies; Vol. 1, No. 2; pp.12-19, 2012, Published by Canadian Centre of Science and Education.

[16] AtiyaParveen, Sobia Habib, Waseem Ahmad, The cloud changing the Indian healthcare system, International Journal of Computer Science and Mobile Computing, IJCSMC, Vol. 2, Issue. 5, May 2013, pg.238 - 243.

[17] VMware Solutions, Your Cloud in Healthcare,Industry brief WHITE PAP ER, 2011, USA. 